\title{
Solar-like oscillations in the G8 V star $\tau$ Ceti $^{\star}$
}

\author{
T. C. Teixeira ${ }^{1,2}$, H. Kjeldsen ${ }^{2}$, T. R. Bedding ${ }^{3}$, F. Bouchy ${ }^{4}$, J. Christensen-Dalsgaard ${ }^{2}$, M. S. Cunha ${ }^{1}$, T. Dall ${ }^{5}$, \\ S. Frandsen ${ }^{2}$, C. Karoff ${ }^{2}$, M. J. P. F. G. Monteiro ${ }^{1,6}$, and F. P. Pijpers ${ }^{2,7}$ \\ ${ }^{1}$ Centro de Astrofísica da Universidade do Porto, Rua das Estrelas, 4150-762 Porto, Portugal \\ e-mail: [mcunha; Mario.Monteiro] @astro.up.pt \\ 2 Department of Physics and Astronomy, Aarhus University, 8000 Aarhus C, Denmark \\ e-mail: [tct; hans; jcd; srf; karoff; fpp] @phys .au.dk \\ 3 Sydney Institute for Astronomy (SIfA), School of Physics, University of Sydney 2006, Australia \\ e-mail: bedding@Physics.usyd.edu.au \\ ${ }^{4}$ Laboratoire d'Astrophysique de Marseille, Traverse du Siphon, BP 8, 13376 Marseille Cedex 12, France \\ e-mail: bouchy@iap.fr \\ 5 Gemini Observatory, 670 N. A'ohoku Pl., Hilo, HI 96720, USA \\ e-mail: tdall@eso.org \\ 6 Departamento de Matematica Aplicada da Faculdade de Ciencias da Universidade do Porto, Portugal \\ 7 Blackett Laboratory, Imperial College London, South Kensington, London SW7 2BW, UK
}

Received 5 August 2008 / Accepted 18 November 2008

\section{ABSTRACT}

\begin{abstract}
We used HARPS to measure oscillations in the low-mass star $\tau$ Cet. Although the data were compromised by instrumental noise, we have been able to extract the main features of the oscillations. We found $\tau$ Cet to oscillate with an amplitude that is about half that of the Sun, and with a mode lifetime that is slightly shorter than solar. The large frequency separation is $169 \mu \mathrm{Hz}$, and we have identified modes with degrees $0,1,2$, and 3 . We used the frequencies to estimate the mean density of the star to an accuracy of $0.45 \%$ which, combined with the interferometric radius, gives a mass of $0.783 \pm 0.012 M_{\odot}(1.6 \%)$.
\end{abstract}

Key words. stars: oscillations - stars: individual: $\tau$ Ceti - stars: individual: $\delta$ Pavonis - stars: individual: $\alpha$ Centauri B

\section{Introduction}

In the past few years, a new generation of high-resolution, highprecision spectrographs has been providing unprecedented opportunities for studying the fine details of stellar interiors and evolution through the detection of tiny stellar oscillations. The observation and analysis of stellar oscillations, or asteroseismology, has the potential to change dramatically our views of stars.

The G8 V star $\tau$ Ceti (HR 509; HD 10700; HIP 8102; $V=$ 3.50 ) is expected to have a convective envelope and therefore to display solar-like oscillations. Since $\tau$ Cet has a lower metallicity than the Sun $([\mathrm{Fe} / \mathrm{H}]=-0.5 \pm 0.03$; Soubiran et al. 1998), it bridges the gap towards very metal-poor population II asteroseismic target stars such as $v$ Ind, where solar-type oscillations have been detected (Bedding et al. 2006; Carrier et al. 2007). Moreover, among stars for which a detection of solar-type oscillations have been attempted (see Bedding \& Kjeldsen 2007; and Aerts et al. 2008, for recent summaries), $\tau$ Cet has the lowest mass.

As a nearby bright star, $\tau$ Cet has been intensively studied. A rotational period of 34 days is suggested by sporadic periodicities in Ca II (Baliunas et al. 1996), but overall $\tau$ Cet is a very inactive star with almost no rotational modulation. This led Gray $\&$ Baliunas (1994) to propose that $\tau$ Cet is seen nearly pole-on, while Judge et al. (2004) have suggested that it may be in a phase analogous to the solar Maunder minimum. Its stability makes it a favoured target for testing the velocity stability of exoplanet

* Based on observations collected at the European Southern Observatory, La Silla, Chile (ESO Programme 74.D-0380). programmes (e.g. Butler et al. 1996). Despite many velocity observations by different groups, no planetary companions have been reported (Wittenmyer et al. 2006). Direct imaging with the Hubble Space Telescope also failed to detect a companion (Schroeder et al. 2000). However, Greaves et al. (2004) have imaged a debris disc around $\tau$ Cet that has a dust mass at least an order of magnitude greater than in the Kuiper Belt.

We note that $\tau$ Cet is particularly suitable for an asteroseismic observing campaign because its radius has been determined interferometrically with an accuracy of $0.5 \%$. The combination of interferometric and asteroseismic results has been applied to several other stars, as discussed in detail by Creevey et al. (2007) and Cunha et al. (2007). As stressed by Brown \& Gilliland (1994), for example, oscillation frequencies are most valuable for testing evolution theories when the other fundamental stellar properties are well-constrained. $\tau$ Cet satisfies this requirement as well as can be done for any single star.

\section{Velocity observations and guiding noise}

We were allocated six nights to observe $\tau$ Cet on 2004 October 2-7, using the HARPS spectrograph (High Accuracy Radial velocity Planet Searcher) on the 3.6-m telescope at the European Southern Observatory on La Silla in Chile. This spectrograph includes a thorium emission lamp to provide a stable wavelength reference.

We obtained 1962 spectra of $\tau$ Cet, with a dead time of $31 \mathrm{~s}$ between exposures. For the first two nights we used an exposure time of $40 \mathrm{~s}$ (resulting in a Nyquist frequency of $7.04 \mathrm{mHz}$ ) but 


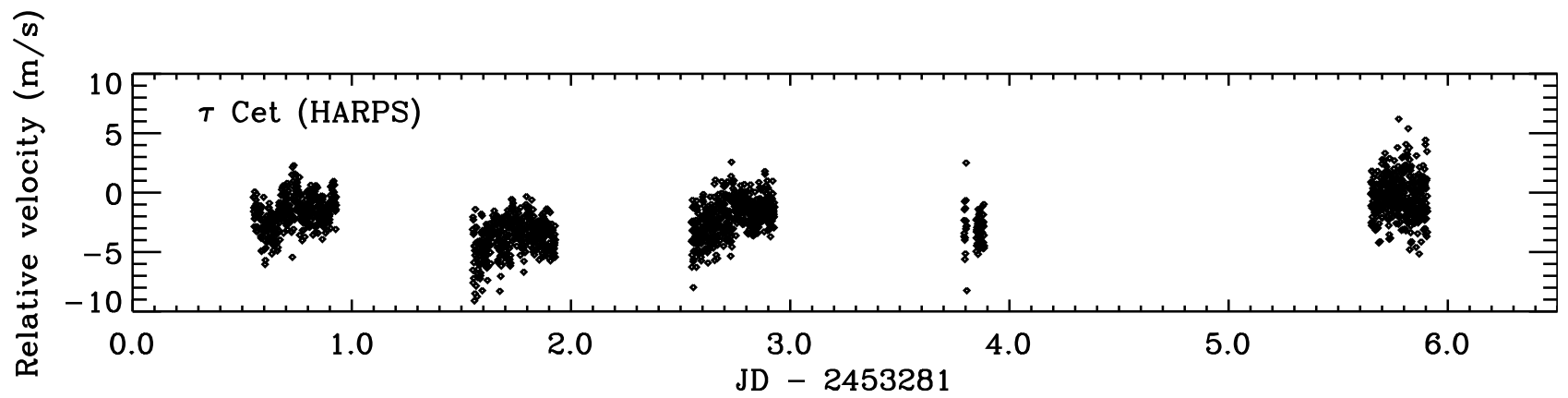

Fig. 1. Time series of velocity measurements of $\tau$ Cet.

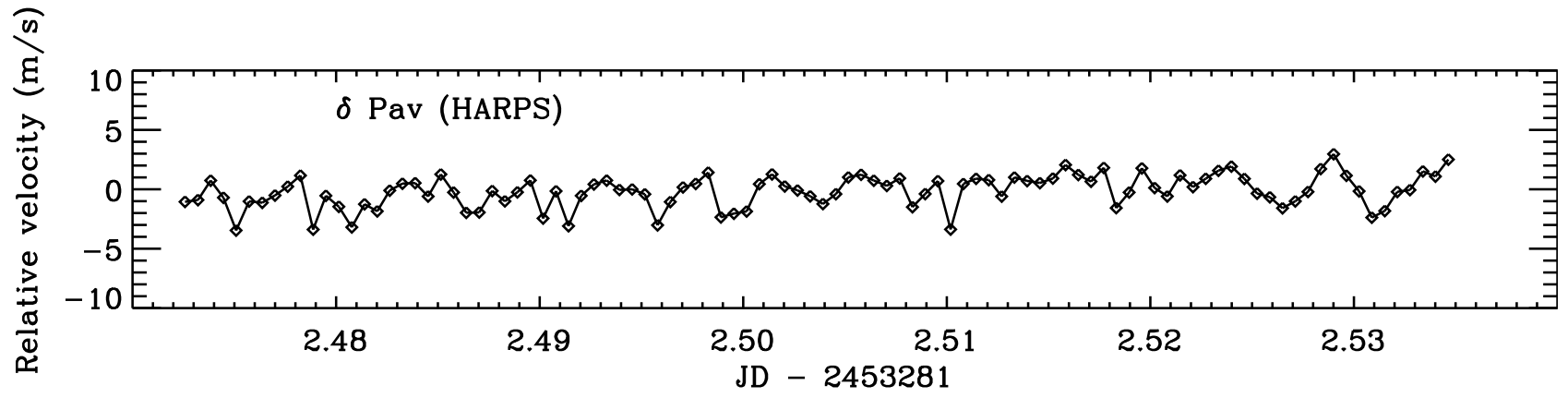

Fig. 2. Velocity measurements of $\delta$ Pav over $1.5 \mathrm{~h}$ at the start of night 3.

shortened this to $23 \mathrm{~s}$ (Nyquist frequency $9.26 \mathrm{mHz}$ ) for the remainder, in order to sample better the noise at high frequencies (see below). The velocities were processed using the method described by Bouchy et al. (2001) and the resulting velocities are shown in Fig. 1. The fourth and fifth nights were mostly lost to poor weather.

For about $1.5 \mathrm{~h}$ at the start of each night, when $\tau$ Cet was inaccessible, we observed the star $\delta$ Pav (HR 7665; HD 190248; HIP 99240; G6-8 IV; $V=3.56$ ). Small amounts of data on this star were also obtained with UVES at the VLT and UCLES at the AAT by Kjeldsen et al. (2005), who found oscillations centred at $2.3 \mathrm{mHz}$ with peak amplitudes close to solar. We obtained a total of 225 spectra of $\delta$ Pav with HARPS, with exposure times of $50 \mathrm{~s}$ (nights 1 and 2) and $23 \mathrm{~s}$ (nights 3 and 5). The velocities for night 3 (100 data points) are shown in Fig. 2.

From the scatter in the velocities and the noise in the power spectra for both $\tau$ Cet and $\delta$ Pav, it was obvious that an unexpected noise source was affecting the velocities. Figure 3 shows the power spectrum for night 3 for both stars. For $\tau$ Cet (upper panel), there is a clear excess at $4 \mathrm{mHz}$, as expected for oscillations in this star. However, there is also a significant power excess around $6 \mathrm{mHz}$. For $\delta$ Pav (lower panel of Fig. 3), the power centred at about $2.2 \mathrm{mHz}$ is from oscillations (see Kjeldsen et al. 2005 ) but we again see additional power at $6 \mathrm{mHz}$. The effect of this instrumental noise is clearly visible by comparing the time series of $\delta$ Pav in Fig. 2 of this paper with that in Fig. 2 of Kjeldsen et al. (2005).

The spurious signal at $6 \mathrm{mHz}$ was later traced to a periodic error in the telescope guiding system. Noise spikes at 3.1 and $6.2 \mathrm{mHz}$ have been reported in HARPS observations of other oscillating stars, namely 70 Oph (Carrier \& Eggenberger 2006), $\alpha$ Cen A (Bazot et al. 2007) and $\beta$ Hyi (Bedding et al. 2007). In the case of $\tau$ Cet, the $6 \mathrm{mHz}$ noise is particularly problematic because it covers a fairly broad range of frequencies and because the stellar oscillations have very low amplitude. There does not seem to be a strong noise signal at $3 \mathrm{mHz}$ in our data, although it is difficult to be certain.

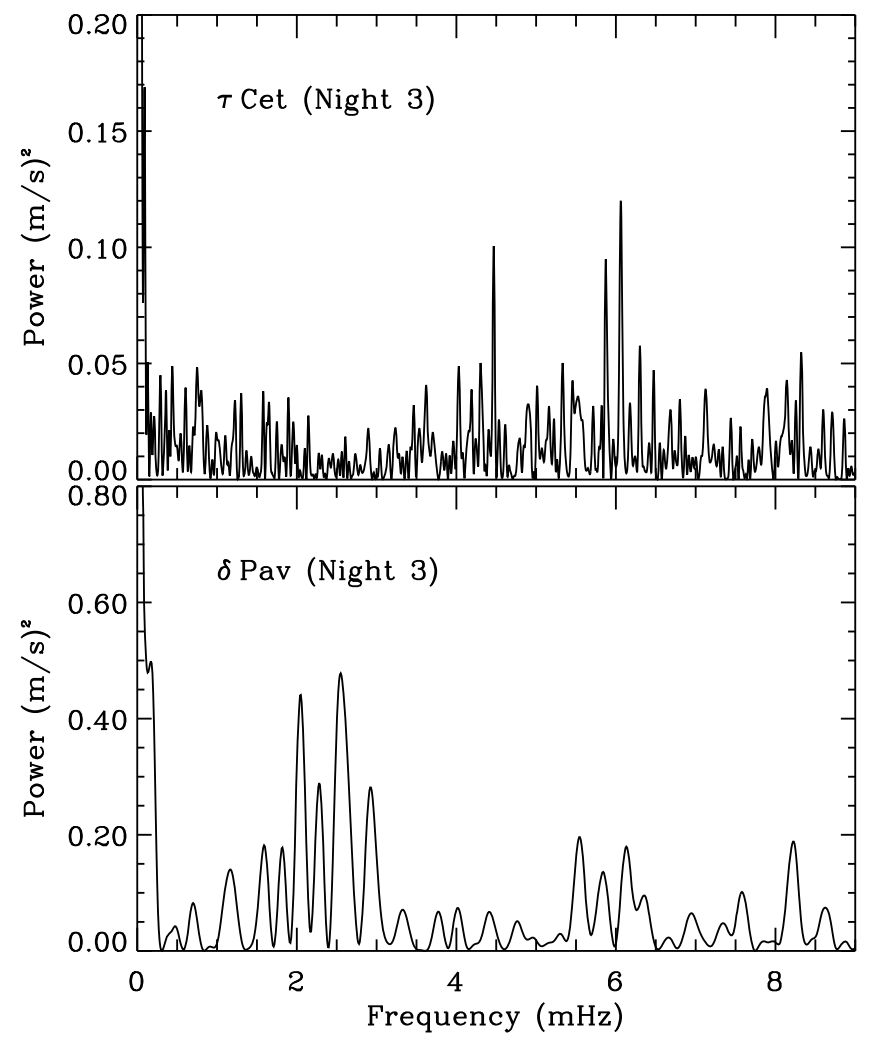

Fig. 3. Power spectrum of velocity measurements for night 3 only, for $\tau$ Cet $(9.1 \mathrm{~h})$ and $\delta$ Pav $(1.5 \mathrm{~h})$.

\section{Data analysis and results}

Our analysis of the velocity data and of the extracted power spectrum for $\tau$ Cet follows the method developed and used for $\alpha$ Cen A (Butler et al. 2004; Bedding et al. 2004), $\alpha$ Cen B (Kjeldsen et al. 2005), $v$ Ind (Bedding et al. 2006) and $\beta$ Hyi 
(Bedding et al. 2007). As usual, we have used the measurement uncertainties, $\sigma_{i}$, as weights in calculating the power spectrum (according to $w_{i}=1 / \sigma_{i}^{2}$ ). The main difference between $\tau$ Cet and other stars that we have analysed, apart from the problem of excess noise from the periodic guiding error, is the single-site nature of the observations. Because of this, we have not attempted to optimize the weights to reduce the sidelobes, in the way that we did for other stars.

\subsection{Adjusting the weights}

As for previous stars, we adjusted the statistical weights to account for bad data points and for night-to-night variations in the noise level. We did this by measuring the noise at frequencies where the oscillation signal and the long-term drifts are negligible. The first step was therefore to remove all power below $800 \mu \mathrm{Hz}$ (to avoid the slow drifts), as well as all power between $3000 \mu \mathrm{Hz}$ and $5500 \mu \mathrm{Hz}$ (which is dominated by the oscillations). This filtering was performed by the standard method of iterative sine-wave fitting (sometimes known as "prewhitening"). In this method, the highest peak is identified in the region of the power spectrum that is to be removed, the corresponding sinusoid is subtracted from the time series, the power spectrum is recomputed and the procedure is repeated until all the power is removed.

Once this was done, the filtered time series for each night was examined for bad data points. These were identified as those deviating from the mean scatter by more than 4- $\sigma$, and were reassigned lower statistical weights. We found that more than $10 \%$ of data points had to be significantly down-weighted. This fraction is much greater than for previous stars that we have observed and indicates the serious effects of the guiding errors. At the same time, we scaled the statistical weights on a night-to-night basis, in order to reflect the noise measured at high frequencies.

The consequence of using the revised weights is a significant improvement in the signal-to-noise, as can be seen in Fig. 4. Comparison of the left and right panels in that figure shows that the adjustment of weights has removed essentially all the excess noise at $6-7 \mathrm{mHz}$ and also decreased the noise level in the range $1-3 \mathrm{mHz}$. The mean noise in those regions, measured in the amplitude spectrum of the whole data set, was reduced from 6.0 to $4.0 \mathrm{~cm} \mathrm{~s}^{-1}$. Most of this reduction came from the down-weighting of bad data points, as described above. Also note that the strongest oscillation peaks are not as high in the combined data (top panel of Fig. 4) as in the shorter subsets (middle and bottom panels). This reflects the finite lifetime of the modes (see Sect. 3.3).

\subsection{The large separation}

The final power spectrum of $\tau$ Cet is shown in the top-right panel of Fig. 4. There is a clear excess due to oscillations which is centred at $4.5 \mathrm{mHz}$. The next step was to search for a regular series of peaks, as expected for p-mode oscillations, and to measure the large frequency separation, $\Delta v$. We did this in two ways. The first was to smooth the power spectrum and then calculate the autocorrelation in the region of excess power, between 2.5 and $6.0 \mathrm{mHz}$. This produced a clear peak at $169 \mu \mathrm{Hz}$.

The second method, which we developed for the Kepler pipeline (Christensen-Dalsgaard et al. 2007), involved measuring the highest peak in the collapsed power spectrum for a range of values of the large separation. The collapsed power spectrum for a given value of $\Delta v$ is calculated by dividing the power spectrum into intervals of length $\Delta v$ and summing these. The result is shown in Fig. 5, and again we see a peak at $169 \mu \mathrm{Hz}$.

\subsection{Oscillations frequencies and mode lifetime}

Mode frequencies for low-degree p-mode oscillations in mainsequence stars are well approximated by a regular series of peaks, with frequencies given by the following asymptotic relation:

$v_{n, l}=\Delta v\left(n+\frac{1}{2} l+\epsilon\right)-l(l+1) D_{0}$.

Here $n$ (the radial order) and $l$ (the angular degree) are integers, $\Delta v$ (the large separation) depends on the sound travel time across the whole star, $D_{0}$ is sensitive to the sound speed near the core and $\epsilon$ is sensitive to the surface layers. See Christensen-Dalsgaard (2004) for a recent review of the theory of solar-like oscillations.

We have extracted the individual oscillation frequencies for $\tau$ Cet using the standard method of iterative sine-wave fitting down to $S / N=2.5$. The single-site nature of the observations and the relatively low signal-to-noise ratio mean that this process is susceptible to one-cycle-per-day ambiguities $( \pm 11.57 \mu \mathrm{Hz})$. On the other hand, we are helped greatly by the fact that both the large and small separations are much greater than the expected mode linewidth, and so all modes are well separated. Furthermore, $\tau$ Cet is an unevolved star and so we expect the oscillation frequencies to follow quite closely the asymptotic relation, without the presence of mixed modes. We have used this information to guide our selection of the correct peaks, but we stress that there is some uncertainty in the correct mode identification.

The extracted frequencies are listed in Table 1. They are also shown in Fig. 6 in echelle format, where the frequencies are stacked in segments of length $\Delta v$. As noted above, the mode identification is uncertain, and this is particularly true for the $l=0$ and $l=2$ modes above $5 \mathrm{mHz}$.

A fit to these frequencies provides the various large and small separations, as listed in Table 2. For the definitions of these separations see Bedding \& Kjeldsen (2003), for example. The separations generally vary with frequency and so the values in Table 2 are given at $4.3 \mathrm{mHz}$. The scatter of these frequencies about smooth ridges in the echelle diagram is about $1-2 \mu \mathrm{Hz}$, which indicates the uncertainties in the individual frequencies in the table. From this scatter we can estimate the mode lifetime, using the method described by Kjeldsen et al. (2005). We find a value of $1.7 \pm 0.5 \mathrm{~d}$, which is slightly lower than the value of $2.88 \pm 0.07 \mathrm{~d}$ measured for the Sun (Chaplin et al. 1997).

\subsection{Stellar parameters}

Detailed fitting of the oscillation frequencies of $\tau$ Cet with theoretical models is beyond the scope of this paper. However, we can use our results to determine the mean density of the star, via the empirical method described by Kjeldsen et al. (2008b). This method corrects the frequencies of stellar models for nearsurface effects by making use of the fact that the offset between observations and models should tend to zero with decreasing frequency. The method involves fitting both $\Delta v$ and the absolute frequencies of the radial modes (i.e., those having degree $l=0$ ). We applied the method to $\tau$ Cet, using models computed with the Aarhus stellar evolution code (ASTEC, Christensen-Dalsgaard 2008a) and the Aarhus adiabatic oscillation package (ADIPLS, Christensen-Dalsgaard 2008b). The result is a mean density for $\tau$ Cet of $2.21 \pm 0.01 \mathrm{~g} \mathrm{~cm}^{-3}(0.45 \%)$. 

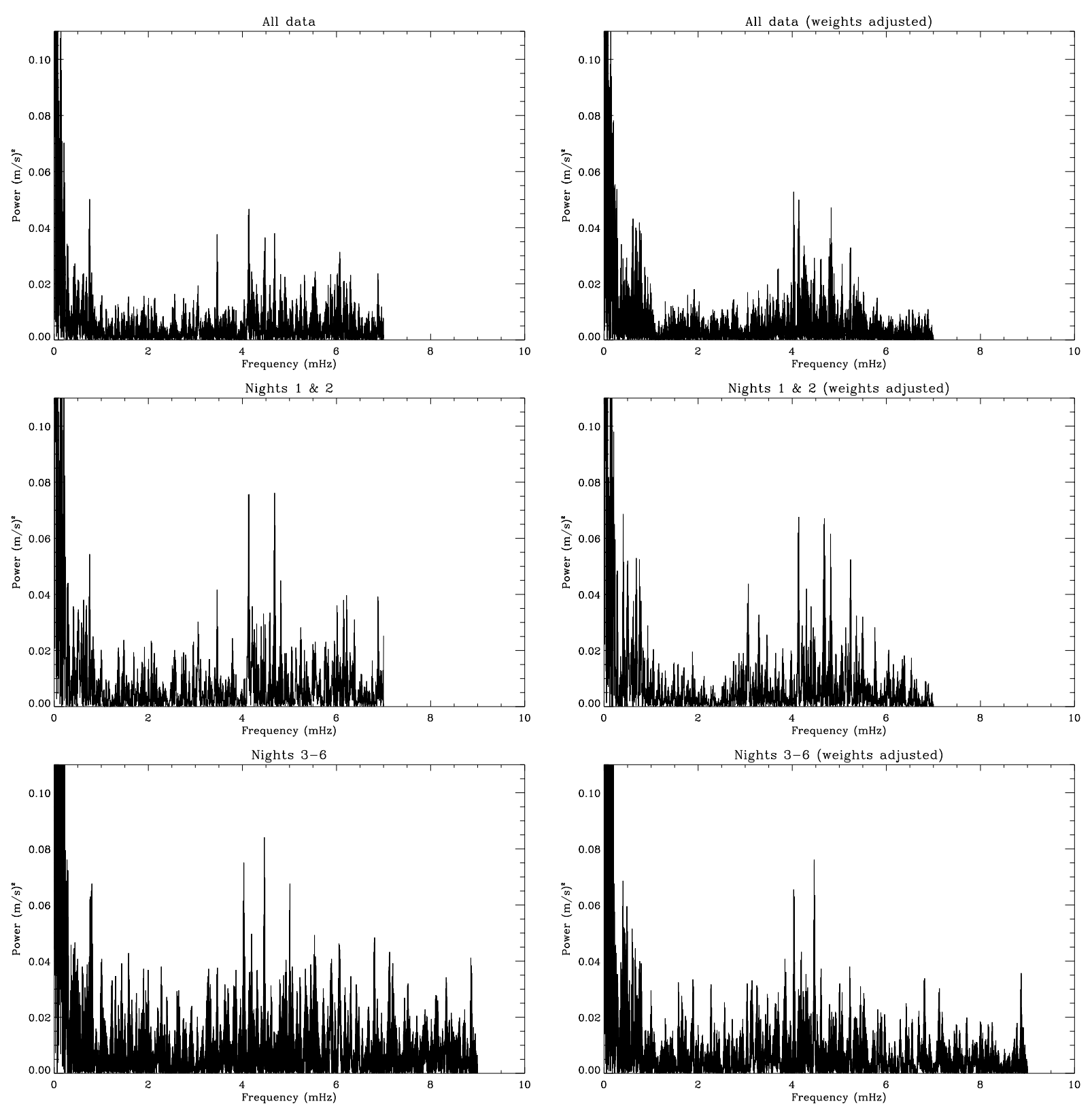

Fig. 4. Power spectrum of $\tau$ Cet for different subsets of the data. The left panels show the spectrum with the use of the raw weights, and the right panel show the spectrum after the weights had been adjusted to account for bad data points and for night-to-night variations in the noise level (see text).

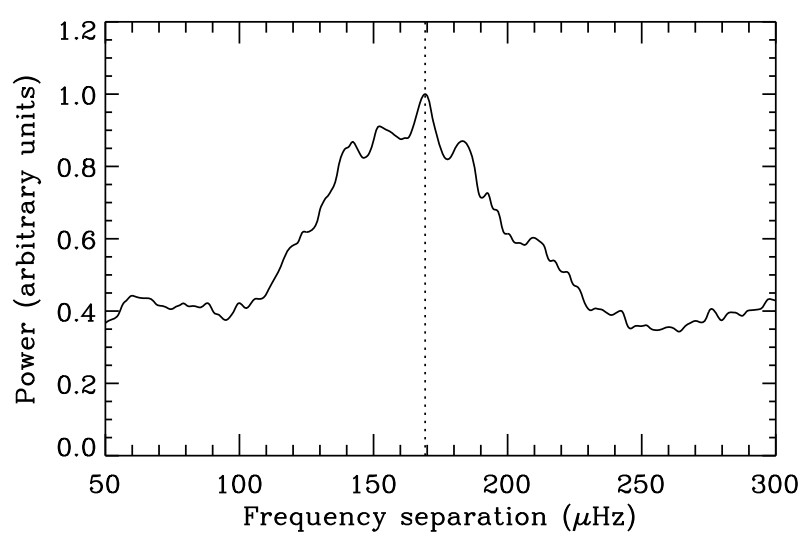

Fig. 5. Summed power of $\tau$ Cet, using different values of the large separation. The dotted line marks the maximum, which occurs at a frequency separation of $169.2 \mu \mathrm{Hz}$.
As mentioned in the Introduction, the angular diameter of $\tau$ Cet has also been measured. This was first done by Pijpers et al. (2003) with the VINCI instrument on the VLTI. They obtained an angular diameter, corrected for limb darkening, of $1.97 \pm 0.05$ mas $(2.5 \%)$, where the uncertainty was dominated by the uncertainty in the angular diameter of the calibrator star. Subsequently, Di Folco et al. (2004) used the VLTI with smaller calibrator stars to obtain an improved diameter of $2.032 \pm 0.031$ mas $(1.5 \%)$. An even more accurate measurement was obtained by Di Folco et al. (2007) using the FLUOR instrument on the CHARA array, giving $2.015 \pm 0.011$ mas $(0.5 \%)$. The weighted mean of these measurements, which we adopt here, is $2.022 \pm 0.010$ mas $(0.5 \%)$. Using the revised Hipparcos parallax for $\tau$ Cet of $273.96 \pm 0.170$ mas (van Leeuwen 2007) gives a radius of $0.793 \pm 0.004 R_{\odot}(0.5 \%)$. Finally, combining this radius with our estimate from asteroseismology of the mean density gives a mass for $\tau$ Cet of $0.783 \pm 0.012 M_{\odot}(1.6 \%)$. 
Table 1. Oscillation frequencies in $\tau$ Cet (in $\mu \mathrm{Hz}$ ).

\begin{tabular}{ccccc}
\hline \hline$n$ & $l=0$ & $l=1$ & $l=2$ & $l=3$ \\
\cline { 2 - 5 } 18 & 3293.4 & & & \\
19 & 3461.7 & & & 3692.9 \\
20 & 3634.5 & & & 3863.7 \\
21 & 3799.3 & 3885.3 & & 4030.3 \\
22 & 3976.1 & 4046.8 & 4126.1 & 4202.5 \\
23 & 4139.9 & 4222.7 & 4298.2 & \\
24 & & 4388.3 & 4469.5 & 4545.1 \\
25 & 4481.4 & & & \\
26 & 4652.3 & & 4811.8 & \\
27 & 4816.2 & 4903.1 & & 5060.5 \\
28 & & 5072.3 & 5151.8 & \\
29 & & 5240.0 & 5317.5 & \\
30 & & 5411.2 & 5492.8 & \\
31 & 5497.9 & & & \\
\hline
\end{tabular}

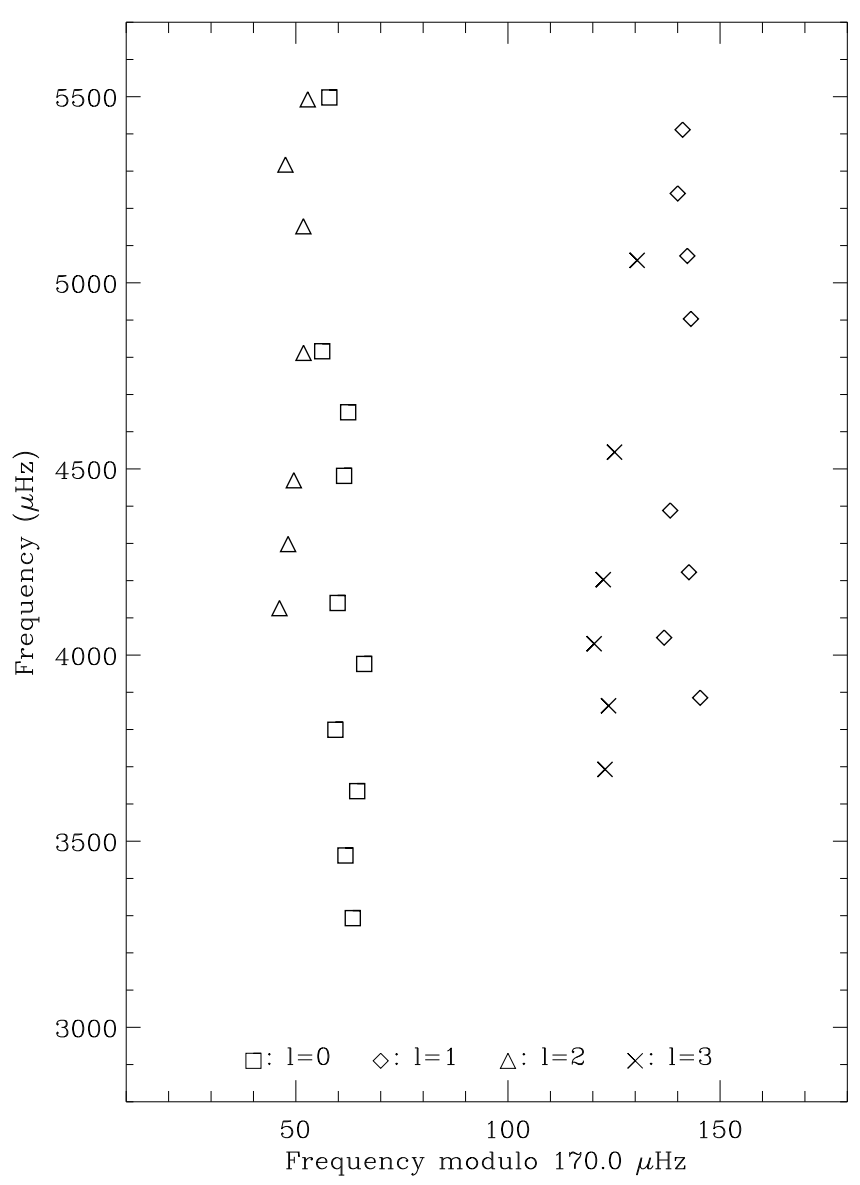

Fig. 6. Echelle diagram of oscillation frequencies for $\tau$ Cet, using the frequencies listed in Table 1.

For convenience, we also give an estimate of the luminosity of $\tau$ Cet. The apparent visual magnitude of $V=3.50 \pm 0.01$, with the revised parallax, gives an absolute magnitude of $M_{V}=5.69 \pm$ 0.01 . Using a bolometric correction for $\tau$ Cet of $-0.17 \pm 0.02$ (Casagrande et al. 2006) and adopting an absolute bolometric magnitude for the Sun of $M_{\mathrm{bol}, \odot}=4.74$ (Bessell et al. 1998), we derive a luminosity for $\tau$ Cet of $0.488 \pm 0.010 L_{\odot}(2.0 \%)$.

\subsection{Oscillation amplitudes}

We have determined the oscillation amplitude per mode from the smoothed power spectrum, using the method described by
Table 2. Oscillation parameters for $\tau$ Cet (see Sect. 3.3).

\begin{tabular}{lr}
\hline \hline Parameter & Value at $4.3 \mathrm{mHz}$ \\
\hline$\Delta v_{0}(\mu \mathrm{Hz})$ & $169.6 \pm 0.2$ \\
$\Delta v_{1}(\mu \mathrm{Hz})$ & $170.0 \pm 0.3$ \\
$\Delta v_{2}(\mu \mathrm{Hz})$ & $170.5 \pm 0.3$ \\
$\Delta v_{3}(\mu \mathrm{Hz})$ & $171.0 \pm 0.3$ \\
$\delta v_{01}(\mu \mathrm{Hz})$ & $4.7 \pm 1.3$ \\
$\delta v_{02}(\mu \mathrm{Hz})$ & $12.7 \pm 1.2$ \\
$\delta v_{03}(\mu \mathrm{Hz})$ & $21.4 \pm 1.2$ \\
$\delta v_{13}(\mu \mathrm{Hz})$ & $16.7 \pm 1.4$ \\
$D_{0}(\mu \mathrm{Hz})$ & $1.77 \pm 0.13$ \\
mode lifetime $(\mathrm{d})$ & $1.7 \pm 0.5$ \\
\hline
\end{tabular}

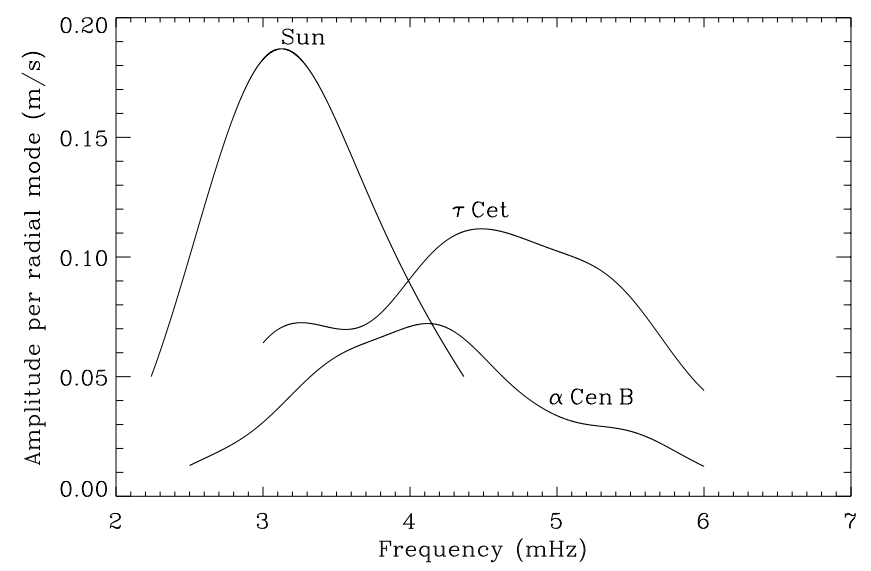

Fig. 7. Smoothed amplitude curve for $\tau$ Cet. For comparison, similar curves are shown for the Sun and $\alpha$ Cen B (Kjeldsen et al. 2008a).

Kjeldsen et al. (2008a). This produces a result that is independent of the stochastic nature of the excitation and damping. The result is shown in Fig. 7. The peak of the envelope occurs at frequency $v_{\max }=4.49 \mathrm{mHz}$ and the peak amplitude per mode (for radial oscillations) is $11.2 \pm 0.8 \mathrm{~cm} / \mathrm{s}$, where the uncertainty is estimated using Eq. (3) of Kjeldsen et al. (2008a). For comparison, we also show in Fig. 7 the amplitude curves measured for the Sun (using stellar techniques) and for $\alpha$ Cen B, both taken from Fig. 8 of Kjeldsen et al. (2008a). Note that the luminosity and mass of $\alpha$ Cen B are, respectively, $0.51 L_{\odot}$ (Y1ldız 2007) and $0.93 M_{\odot}$ (Pourbaix et al. 2002). With this in mind, we see that the relative amplitudes of the three stars in Fig. 7 are in reasonable agreement with the $L / M$ scaling relation proposed by Kjeldsen \& Bedding (1995; see also Samadi et al. 2007)

\section{Conclusions}

We have used HARPS to measure oscillations in the low-mass star $\tau$ Cet. Although the data were compromised by instrumental noise, we have been able to extract the main features of the oscillations. We found $\tau$ Cet to oscillate with an amplitude that is about half that of the Sun, and with a mode lifetime that is slightly smaller than solar. The large frequency separation is $169 \mu \mathrm{Hz}$, and we have identified modes with degrees $0,1,2$ and 3 . It is important to stress that, given the relatively low signal-to-noise ratio and the single-site nature of the observations, there is some uncertainty in the correct mode identification.

We used the frequencies of the radial modes to estimate the mean density of the star to an accuracy of $0.45 \%$, from which we derived a mass of $0.783 \pm 0.012 M_{\odot}(1.6 \%)$. More detailed 
modelling of the oscillation frequencies will be the subject of a future paper.

Acknowledgements. We thank the referee for helpful suggestions. This work has been supported by the Danish Natural Science Research Council and the Australian Research Council. M.C. is supported by the Ciencia2007 Programme from FCT (C2007-CAUP-FCT/136/2006).

\section{References}

Aerts, C., Christensen-Dalsgaard, J., Cunha, M., \& Kurtz, D. W. 2008, Sol. Phys., 251, 3

Baliunas, S. L., Nesme-Ribes, E., Sokoloff, D., \& Soon, W. H. 1996, ApJ, 460, 848

Bazot, M., Bouchy, F., Kjeldsen, H., et al. 2007, A\&A, 470, 295

Bedding, T. R., \& Kjeldsen, H. 2003, PASA, 20, 203

Bedding, T. R., \& Kjeldsen, H. 2007, in Unsolved Problems in Stellar Physics:

A Conference in Honour of Douglas Gough, ed. R. J. Stancliffe, G. Houdek,

R. G. Martin, \& C. A. Tout, AIP Conf. Proc., 948, 117

Bedding, T. R., Kjeldsen, H., Butler, R. P., et al. 2004, ApJ, 614, 380

Bedding, T. R., Butler, R. P., Carrier, F., et al. 2006, ApJ, 647, 558

Bedding, T. R., Kjeldsen, H., Arentoft, T., et al. 2007, ApJ, 663, 1315

Bessell, M. S., Castelli, F., \& Plez, B. 1998, A\&A, 333, 231; erratum: 337, 321

Bouchy, F., Pepe, F., \& Queloz, D. 2001, A\&A, 374, 733

Brown, T. M., \& Gilliland, R. L. 1994, ARA\&A, 32, 37

Butler, R. P., Marcy, G. W., Williams, E., et al. 1996, PASP, 108, 500

Butler, R. P., Bedding, T. R., Kjeldsen, H., et al. 2004, ApJ, 600, L75

Carrier, F., \& Eggenberger, P. 2006, A\&A, 450, 695

Carrier, F., Kjeldsen, H., Bedding, T. R., et al. 2007, A\&A, 470, 1059
Casagrande, L., Portinari, L., \& Flynn, C. 2006, MNRAS, 373, 13

Chaplin, W. J., Elsworth, Y., Isaak, G. R., et al. 1997, MNRAS, 288, 623

Christensen-Dalsgaard, J. 2004, Sol. Phys., 220, 137

Christensen-Dalsgaard, J. 2008a, Ap\&SS, 316, 13

Christensen-Dalsgaard, J. 2008b, Ap\&SS, 316, 113

Christensen-Dalsgaard, J., Arentoft, T., Brown, T. M., et al. 2007, Commun. Asteroseismol., 150, 350

Creevey, O. L., Monteiro, M. J. P. F. G., Metcalfe, T. S., et al. 2007, ApJ, 659, 616

Cunha, M. S., Aerts, C., Christensen-Dalsgaard, J., et al. 2007, A\&AR, 14, 217

Di Folco, E., Thévenin, F., Kervella, P., et al. 2004, A\&A, 426, 601

Di Folco, E., Absil, O., Augereau, J.-C., et al. 2007, A\&A, 475, 243

Gray, D. F., \& Baliunas, S. L. 1994, ApJ, 427, 1042

Greaves, J. S., Wyatt, M. C., Holland, W. S., \& Dent, W. R. F. 2004, MNRAS, 351, L54

Judge, P. G., Saar, S. H., Carlsson, M., \& Ayres, T. R. 2004, ApJ, 609, 392

Kjeldsen, H., \& Bedding, T. R. 1995, A\&A, 293, 87

Kjeldsen, H., Bedding, T. R., Butler, R. P., et al. 2005, ApJ, 635, 1281

Kjeldsen, H., Bedding, T. R., Arentoft, T., et al. 2008a, ApJ, 682, 1370

Kjeldsen, H., Bedding, T. R., \& Christensen-Dalsgaard, J. 2008b, ApJ, 683, L175

Pijpers, F. P., Teixeira, T. C., Garcia, P. J., et al. 2003, A\&A, 406, L15

Pourbaix, D., Nidever, D., McCarthy, C., et al. 2002, A\&A, 386, 280

Samadi, R., Georgobiani, D., Trampedach, R. R., et al. 2007, A\&A, 463, 297

Schroeder, D. J., Golimowski, D. A., Brukardt, R. A., et al. 2000, AJ, 119, 906

Soubiran, C., Katz, D., \& Cayrel, R. 1998, A\&AS, 133, 221

van Leeuwen, F. 2007, Hipparcos, the New Reduction of the Raw Data (Dordrecht: Springer)

Wittenmyer, R. A., Endl, M., Cochran, W. D., et al. 2006, AJ, 132, 177

Yildı, M. 2007, MNRAS, 374, 1264 